\title{
Reducing Interference in Ad hoc Networks through Topology Control
}

\author{
Tomas Johansson \\ EISLAB \\ Luleå University of Technology \\ SE-971 87 Luleå, Sweden \\ tomasjo@sm.luth.se
}

\author{
Lenka Carr-Motyčková \\ EISLAB \\ Luleå University of Technology \\ SE-971 87 Luleå, Sweden \\ lenka@sm.luth.se
}

\begin{abstract}
Topology control aims to increase the lifetime of an ad hoc network by selecting only a subset of the available links to be used for routing. The tradeoff between keeping the spanner properties of the graph while sparsifying the graph has been well studied. However, it has often been assumed that a sparse graph implicitly has low interference, but recent research shows that that is not necessarily true. In this paper, we discuss different methods to measure interference, and present a new interference model that aims to describe the interference of the entire network, rather than just the worst part of it.

We present API, a topology control algorithm that serves two purposes: it minimizes the interference in the network according to our metrics, and it keeps the spanner properties of the original graph. The paper is completed by simulations that compare different topologies with respect to different interference metrics.
\end{abstract}

\section{Categories and Subject Descriptors}

C.2.4 [Computer-Communication Networks]: Distributed Systems

\section{General Terms}

Algorithms

\section{Keywords}

Ad hoc networks, topology control, interference

\section{INTRODUCTION}

In a wireless ad-hoc network, physical constraints often force the individual nodes to use a battery as power source. Therefore, energy is the factor that limits the lifetime of the network.

Permission to make digital or hard copies of all or part of this work for personal or classroom use is granted without fee provided that copies are not made or distributed for profit or commercial advantage and that copies bear this notice and the full citation on the first page. To copy otherwise, to republish, to post on servers or to redistribute to lists, requires prior specific permission and/or a fee.

DIALM-POMC'05, September 2, 2005, Cologne, Germany.

Copyright 2005 ACM 1-59593-092-2/05/0009 ...\$5.00.
One way to reduce the power consumption and extend the lifetime of the network is by topology control, a method to choose a suitable topology to be used for routing in the network. This is done by selecting a subset of the available links in the network graph $G=(V, E)$ to form the reduced graph $G_{T C}=\left(V, E_{T C}\right)$. The general approach of a topology control algorithm is to remove longer links from the network in order to force the nodes to use several shorter hops instead, using a smaller amount of energy. On the other hand, if too many edges (or a wrong selection of edges) are removed, the paths become unacceptably long with respect to the number of hops, and the network may even become disconnected.

In addition to the obvious requirement that the reduced graph must be connected, a stronger requirement is that it has to be a spanner. A $t$-spanner (where $t$ is a constant) is a graph where the shortest path in $G_{T C}$ between any two nodes is at most $t$ times longer than the shortest path between these nodes in $G$. A graph can be a spanner with respect to the euclidian distance as well as with respect to the energy cost of the path. Another common requirement, in order to reduce interference, is that the reduced graph should be sparse: that the number of edges should be in the order of the number of nodes. A stronger version of that requirement is that the maximum degree (number of neighbors, of any node) should not exceed a given constant. A graph that fulfills that requirement must also be sparse, but a sparse graph does not necessarily fulfill the maximum degree requirement.

Transmitting nodes influence the ability of other nodes to receive data. A node is not able to receive data from its neighbor if another neighbor is transmitting at the same time. This mutual disturbance of communication is called interference. Reducing interference in the network leads to fewer collisions and packet retransmissions, which indirectly reduces the power consumption and extends the lifetime of the network. Therefore, reducing the interference in the reduced graph $G_{T C}$ is an important goal for topology control algorithms. As mentioned earlier, previous work in topology control often assumes that a sparse network implies low interference. However, it has been shown in [3] that a low node degree does not guarantee low interference. Interference is therefore a factor that must be specifically adressed when working in the field of topology control.

In this paper we present new metrics for the interference of a given graph, and relate it to metrics used by other 
authors. We also present a topology control algorithm that minimizes the average path interference of a graph while still preserving the spanner property.

The paper is organized as follows: Section 2 presents an overview of previous work on topology control algorithms, especially concerning interference. Several different metrics for interference are covered. In section 3, we present our network model that will be used in this paper as well as a survey of metrics used by other authors. The section is concluded by definitions of new metrics for interference in an ad-hoc network that we introduce. Section 4 discusses how different interference models give different results when aiming to reduce interference in a network. In section 5 , we describe an algorithm that minimizes the interference in the network according to our metric while keeping the spanner properties of the original graph. In section 6 we compare this algorithm with other topology control algorithms using simulations and discuss properties of different topologies with respect to different interference metrics. Finally, section 7 concludes our work.

\section{RELATED WORK}

Earlier topology control algorithms were often based on computational geometry structures, such as the minimum spanning tree [8], or the Delaunay triangulation [5]. In [9], Rodoplu and Meng present an algorithm that keeps all energy optimal paths. Their topology, which takes an energy model as input, is a general version of the Gabriel graph[4]. The Gabriel graph of a set of vertices in the plane is defined as follows: the edge $(p, q)$ exists iff the circle with diameter $p q$ does not contain any vertice other than $p$ or $q$ in its interior. If the energy model used as input has the cost for a given distance $d$ to be Energy $=O\left(d^{2}\right)$, the structure produced by the algorithm in [9] is the Gabriel graph. However, the Gabriel graph is not an Euclidian spanner: it was shown in [1] that the Euclidian spanning ratio for Gabriel graphs is $\Theta(\sqrt{n})$ in the worst case. The XTC topology control algorithm [13] is shown to produce a subgraph of the relative neighborhood graph [10]: in both algorithms, an edge between nodes $u$ and $v$ cannot exist if a node $w$ exists such that $|u v|>\max (|u w|,|v w|)$. However, unlike the $\mathrm{RNG}$, the XTC algorithm can also remove the edge $(u, v)$ if $|u v|=\max (|u w|,|v w|)$.

In general, topology control algorithms do not deal directly with reducing the interference. Instead, it is assumed that a sparse graph will lead to low interference. For example, [12] assumes that a low node degree in the graph implies low interference. However, in [3] it is shown that even a graph with a maximum degree of 2 can have a very high interference relative to the optimal solution. This is because a node can interfere with other nodes that are not direct neighbors in the graph. Even the nearest neighbor forest topology, where each node only connects to its nearest neighbor, does not guarantee low interference. Thus, the only way to guarantee low interference is to define how to measure it and design an algorithm that explicitly reduces interference. There is, however, a tradeoff between low interference and connectivity: as links are removed to reduce interference, the paths in the network grow in length which leads to higher delay and power consumption.

[2] examines the trade-off between congestion, dilation, and power consumption. The paper defines the congestion of a network as the maximum congestion an edge experiences; the congestion for a single edge $e$ is the sum of the load on $e$ and the load of all the edges that interfere with $e$. The dilation of a network is defined as the length of the longest path in the network. It is shown that $\Omega(W)$, where $W$ is the amount of traffic in the network, is a lower bound for the congestion value multiplied by the dilation of that network. Also, it is shown that it is impossible to optimize both the congestion and the energy efficiency of a network: one of the factors will be at least a polynomial factor worse than in the optimal network.

An important question is how the amount of interference in a network should be measured. [3] presents a trafficindependent model of the amount of interference in a network, where the interference of the entire network is defined as the maximum edge coverage: the maximum number of nodes affected by one specific link in the network. The authors show that it is impossible for a local algorithm to always find the topology that gives the lowest maximum edge coverage, since knowledge of the entire network is needed. However, they present the algorithm LISE (Low Interference Spanner Establisher) that solves a similar problem: find the graph with the lowest possible maximum edge coverage that also is a t-spanner, where $t$ is a constant that can be chosen freely. As long as every edge (or in practice, one of its incident nodes) has knowledge of its $\frac{t}{2}$-neighborhood ${ }^{1}$, the path with the lowest maximum edge coverage that fulfills the spanner requirement can be computed locally. A distributed version of the algorithm (Local LISE, or LLISE) is also described. This work is expanded upon in [11], where an alternative, receiver-centric, interference model is introduced. In this model, the coverage of a node $v$ in the network graph $G$ is defined as the number of nodes covering $v$ with their disks induced by their transmission ranges set to reach their farthest neighbor in $G$. The interference of the entire network is defined by the maximum coverage for any node in the network. One drawback of these models is that they do not consider the interference of entire paths, but instead aim to reduce the interference locally. This can lead to longer paths, which in turn leads to a larger number of nodes being affected by the communication. In [7] an alternative interference metric that corresponds to the average interference of the entire network is presented: the interference is defined as the sum of the edge coverage of all edges in the network, divided by the number of nodes in the network.

A different interference model is presented in [6]. This metric also takes the transmission power into account: if the number of neighbors is constant when a node increases or decreases its transmission power level from $P_{1}$ to $P_{2}$, the interference measure should increase/decrease as well. Also, if two nodes $N_{1}$ and $N_{2}$ are using the same transmission power level but have different numbers of neighbors, the interference measures of the two nodes should differ to reflect the difference in the number of neighbors.

\footnotetext{
${ }^{1}$ The $t$-neighborhood of an edge $e$ is defined as all edges in the graph that can be reached by a path of length no more than $t|e|$, starting from one of $e$ 's incident nodes.
} 


\section{MODEL}

\subsection{Network representation}

In this paper, an ad hoc network is modelled as an Euclidian graph $G=(V, E)$ with the vertices in $V$ representing network nodes, and the edges $E$ representing communication links. The euclidian position of the vertices in the graph corresponds to the physical position of the nodes in the euclidian two dimensional space, which means that the edge weight $w(u, v)$ represents the physical distance between nodes $u$ and $v$. Each node $u$ has a maximum transmission range $R_{u}$. Since we only consider undirected links, a link $u v$ can only exist if the distance between the nodes $u$ and $v$ is no larger than $\min \left(R_{u}, R_{v}\right)$.

We assume that any node can adjust its transmission power to any value from 0 to its maximum transmission power, depending on the desired transmission radius: when transmitting to node $v$, node $u$ uses the lowest possible transmission power needed to reach $v$. A common path loss model says that the signal strength received by a node can be described as $p / d^{\alpha}$, where $p$ is the transmission power used by the sending node, and $d$ is the distance between two nodes. $\alpha$ is a path loss gradient, depending on the transmission environment. Consequently, the energy cost $c(u, v)$ to send a message of fixed length directly from node $u$ to node $v$ is $\Theta\left(|u, v|^{\alpha}\right)$. The energy cost of a path is defined as the sum of the energy costs of all edges in the path.

\subsection{Existing interference metrics}

Topology control algorithms that aim to reduce interference are based on different metrics, that are used to minimize the interference. Unless the traffic pattern in a network is known in advance, the amount of interference in the network can only be based on the network topology. In [3], the interference of a network is defined as the maximum edge coverage occuring in the network, where the coverage of an edge $e=(u, v)$ is defined as the number of nodes that are within distance $|u, v|$ to at least one of $u$ and $v$, or more formally:

$$
\operatorname{Cov}(e)=|\{w \in V,|v, w| \leq|u, v| \cup|u, w| \leq|u, v|\}|
$$

and

$$
\text { MaxEdgeCoverage }(G)=\max _{e \in E} \operatorname{Cov}(e)
$$

In the subsequent article [11] the authors claim that the previous definition is problematic, since it is sender-centric, and since small changes in the network can drastically change the interference measure. Instead, they propose to define interference of a graph as the maximum interference of any node in the graph. The interference of a node $v$ is defined as the number of nodes in the graph that cover $v$ with their transmission disks when they communicate with their farthest neighbor in the graph. More formally, if $u_{r}$ is the distance between node $u$ and its farthest neighbor in $G$, we have:

$$
\operatorname{Cov}(v)=\left|\left\{u|u \in V v,| u, v \mid<u_{r}\right\}\right|
$$

and

$$
\text { MaxNodeCoverage }(G)=\max _{v \in V} \operatorname{Cov}(v)
$$

Both the described approaches work according the same principle: the global interference in a network depends solely on the local part with the highest interference. Reducing the interference in that part by definition reduces the interference of the entire network. One problem is that the metrics do not consider the interference in general; a network with high interference in one place and low interference everywhere else could have the same interference as another network with equally high interference everywhere. In [7], the authors extend the work in [3] by defining average edge interference as the sum of the coverage of all edges in the graph, divided by the number of nodes in the graph:

$$
\text { AverageEdgeCoverage }(G)=\left(\sum_{e \in E} \operatorname{Cov}(e)\right) /|V|
$$

Using this definition, a small change in the topology will not change the interference measure as much as it could using the definition in [3].

\subsection{Proposed new metrics}

However, this definition does not take into account the length of the paths in the graph. This means that the paths can grow indefinitely without affecting the interference metric. Therefore, we define the average path interference of a graph as the sum of interference for all interference-optimal paths between node pairs, divided by the number of all node pairs in the graph. The interference-optimal path between nodes $u$ and $v$ is the path $\operatorname{Iopt}_{u v}=\left\{e_{1}, e_{2}, \ldots, e_{k}\right\}$ between $u$ and $v$ that has the lowest interference, according to the following definition: the interference of a path is defined as the sum of the coverage of all edges in the path, according to the definition of edge coverage in [3].

$$
\operatorname{TotIoptPI}(G)=\sum_{u, v \in V} \sum_{e \in I o p t P_{u v}} \operatorname{Cov}(e)
$$

If the resulting value is divided by the total number of node pairs that are connected in $G$, we will get the average interference for all minimum-interference paths.

A metric that considers the interference when shortestpath routing is used can be obtained by replacing the interference-optimal path Iopt $P_{u v}$ with the shortest path $S P_{u v}$, with the rest of the formula identical:

$$
\operatorname{TotSPI}(G)=\sum_{u, v \in V} \sum_{e \in S P_{u v}} \operatorname{Cov}(e)
$$

The average interference for all shortest paths is defined as $\operatorname{TotSPI}(\mathrm{G})$ divided by the number of node pairs connected in $G$.

An alternate way to view this metric is to view it as it assigns a weight to every edge, based on the usage of that edge assuming shortest-path routing and uniform traffic among all nodes. This means that the coverage of certain edges have a larger impact on the total graph interference than other edges. It is worth noting that even if the average edge coverage of one graph $G_{1}=\left(V, E_{1}\right)$ is lower than another graph $G_{2}=\left(V, E_{2}\right)$, graph $G_{2}$ can still have lower interference according to this metric.

Every topology control algorithm removes some edges from the original graph $G$ in order to reduce the energy consumption of the network. In the new topology, the interference-optimal with respect to $G$ may disappear and be replaced by a different path that is interference-optimal with respect to $G_{T C}$. Then it is important to evaluate how 
much worse is the interference-optimal paths in $G_{T C}$ than in $G$. The maximum interference difference metric is defined as the biggest difference in interference between Iopt $P_{u v}$ in the original graph compared to Iopt $P_{u v}$ in the graph that is the output of a topology control algorithm. More formally:

$$
M D\left(G_{T C}\right)=\max _{u, v \in V}\left(\operatorname{Iopt} P_{u v} I\left(G_{T C}\right)-\operatorname{Iopt} P_{u v} I(G)\right)
$$

where $\operatorname{Iopt}_{u v} I(G)$ is the interference of the interferenceoptimal path between nodes $u$ and $v$ in $G$.

\section{COMPARISON OF INTERFERENCE METRICS}

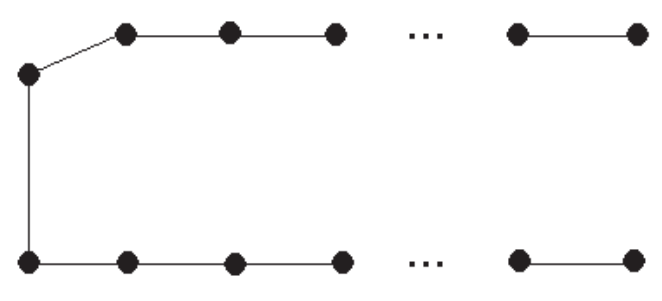

Figure 1: Example topology.

In order to reduce interference, it is important to consider the metric that is used. Different metrics can give vastly different results for the same topologies: a topology that has low interference according to one metric might be far from optimal considering another metric.

Figure 1 shows the topology that gives the lowest interference according to the max edge coverage metric ([3]), while still connecting the entire network. Since the upper and lower group of nodes have to be connected, the leftmost connection is the one which results in the lowest interference. However, this means that a message between any node in the top half and any node in the bottom half of the topology has to use the leftmost edge. Using the average path interference metric, the interference for the graph would be $\Theta(n)$.

\section{PRODUCING A LOW-INTERFERENCE SPANNER}

This section describes a topology control algorithm that creates a reduced graph $G_{T C}=\left(V, E_{T C}\right)$ that aims to reduce the interference according to our definition of average path interference $(2,3)$, while still retaining energy spanner properties. Energy cost $c(u, v)$ as defined in section 3 is used as a measure of a connection quality between two nodes.

\subsection{Algorithm description}

The Average Path Interference (API) algorithm consists of two steps: computing a Gabriel graph, and reducing the graph. Calculating the Gabriel graph is done in the following way:
For each node $u$ :

1. Broadcast a test message with maximum signal strength.

2. For each received test message:

2a. Denote the received signal strength from $v$ as $p_{r e c}^{v}$.

2b. Evaluate the distance $d$

from node $u$ to neighbor $v$, using the

signal degradation model

$p_{\text {rec }}=p_{\text {org }} / d^{\alpha}$.

2c. Calculate the energy cost $c(u, v)$

needed for sending a message from $u$ to $v$

based on the cost function $c(u, v)=d^{\alpha}$.

2 d. Respond to $v$ with the value $c(u, v)$.

3. Wait until all neighbors responds to $u$ with $c(v, u)$, where $v$ is the neighbor.

4. Broadcast to every neighbor the value of

$c(v, u)$ for every neighbor $v$.

5 . For each neighbor $v$ :

Activate link $(u, v)$ iff no node $w$ exists

such that $c(u, w)+c(w, v) \leq c(u, v)$.

The next step is to remove links that lead to high interference. The coverage of each link can be calculated locally. If a link can be replaced with two links that together have smaller coverage, it is removed. In practice, the algorithm marks the links that will be used and all the unmarked links are removed.

For each node $u$ :

For each neighbor $v$ :

If there exists a node $w$ such that $\operatorname{Cov}(u, w)+\operatorname{Cov}(w, v)<\operatorname{Cov}(u, v)$, mark $(u, w)$ and $(w, v)$ to retain. Otherwise, mark $(u, v)$ to retain.

Remove all unmarked links.

\subsection{Analysis}

The graph produced by the algorithm is an energy spanner.

Proof. Since the Gabriel graph that is produced in the first step of the algorithm contains all energy-optimal paths, we only need to consider the second step.

When a link $e$ is deleted, the two links $e_{1}, e_{2}$ that replace it cannot both be longer than the removed link. If they were, their combined coverage would have to be larger than the coverage of the removed link. This is because the nodes that were covered by $e$ according to (1) are covered by either $e_{1}$ or $e_{2}$ if they both are longer than $e$. If the combined coverage by $e_{1}$ and $e_{2}$ is not smaller than the coverage of $e$, $e$ would not have been replaced in the first case. This leads to a contradiction.

If the distance of the original link is $d$ and the distances of the two links that replace it are $d_{1}$ and $d_{2}$, we know that $d_{1} \leq d+d_{2}$, and conversely for $d_{2}$. Since at least one of $d_{1}$ and $d_{2}$ is at most $d$, the other replacing link can be at most $d+d=2 d$. Therefore, if the original length was $l$ and the original energy cost was $l^{\alpha}$ (where $\alpha$ is a constant depending on the energy model), the new energy cost will be no larger than $l^{\alpha}+(2 l)^{\alpha}$. In other words, the ratio between the new energy cost and the old is $1+2^{\alpha}$. 

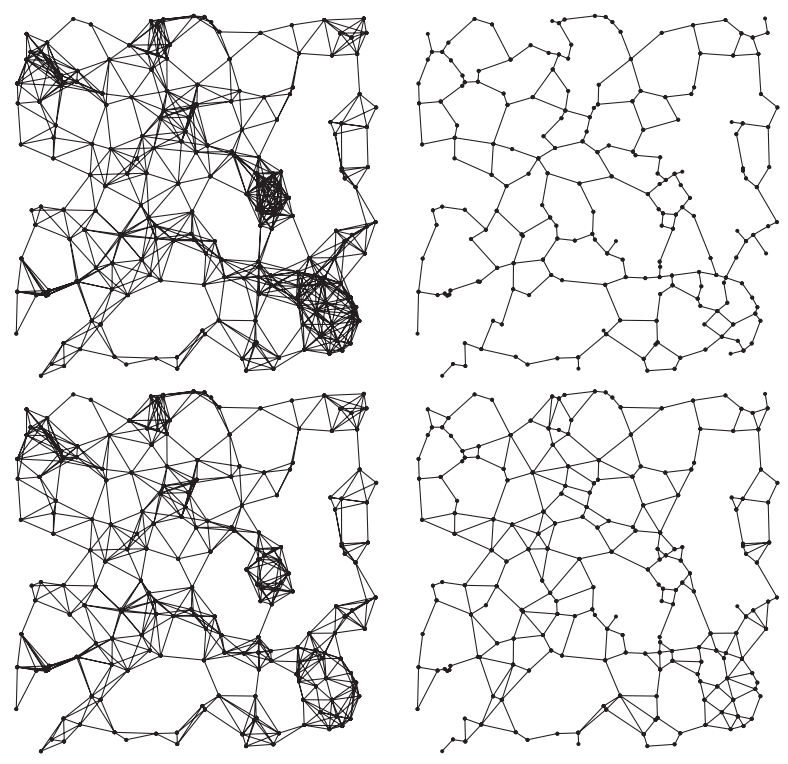

Figure 2: The figures show the results of three different topology control algorithms being performed on the original topology (upper left): the XTC algorithm (upper right), the LISE algorithm (lower left), and finally the API algorithm (lower right).

Since the Gabriel graph is planar, the reduced Gabriel graph produced by the algorithm wil be planar as well.

The API algorithm is not guaranteed to produce the optimal graph with respect to interference, since each node only has local knowledge. However, edges that can be replaced with two other edges in order to reduce interference are removed from the graph. The algorithm may miss edges that can be replaced with longer paths, but as the length of a path grows the total interference of the path is likely to grow as well, and the path is less likely to be a candidate to replace an edge.

\section{SIMULATIONS}

In this section we present the interference properties of topologies produced by three different topology control algorithms (see Figure 2). The topologies were produced by placing nodes randomly and uniformly on a square field of size 20 by 20 units, where all nodes had the common communication range of 2,5 units. A link between two nodes exists if and only if the distance between them is less than the range. The networks were generated using different number of nodes in order to create networks of various density. For every density, three different topologies were generated. On each topology, the different topology control algorithms were run. The values presented here are mean values of the three different topologies for each density.

\subsection{Topology control algorithms}

\subsubsection{Low Interference Spanner Establisher (LISE)}

The LISE algorithm [3] aims to reduce the edge coverage in the network, with the requirement that the resulting graph must be an euclidian $t$-spanner. This means that an edge $e$ with the length $l$ can be removed if there exist an alternate path no longer than $t l$, where no edge has more coverage than $e$. The larger $t$ is, the longer the replacement paths can be. This can lead to longer delays, as well as more coverage if you take the entire replacement path into account.

The time complexity of LISE is $O(t)$, since each node needs to collect information about its $t / 2$-neighborhood. If the network is mobile, and the topology needs to be updated continuously, the time complexity can be an important factor. Also, the increased message complexity means that this algorithm will require more energy than topology control algorithms that only use local knowledge.

In the simulations the $t$-value was set to 4 , since simulation results in [3] indicates that it is a good compromise between reducing interference and keeping the length of the paths as low as possible.

\subsubsection{XTC}

Unlike LISE, in the XTC algorithm [13] each node only needs local knowledge. Given a unit disk graph $G$, the corresponding $G_{X T C}$ graph is planar and have a degree of at most 6 , since no two adjacent edges in $G_{X T C}$ can enclose an angle less than $\pi / 3$. Also, for any weighted graph $G^{\prime}$ the corresponding $G_{X T C}^{\prime}$ does not contain any cycles of length 3 ; in other words, the girth of $G_{X T C}^{\prime}$ is at least 4 . The message complexity of XTC is $O(\Delta)$, where $\Delta$ is the maximum degree of the network. This i because each node must contact all its neighbors. The time complexity is $O(1)$, since only communication between neighboring nodes is needed.

\subsubsection{API}

As XTC, the API algorithm only uses local information. The resulting graph is a reduced version of the Gabriel graph, where edges that have higher coverage than their neighbors are removed. The resulting graph is an energy spanner, though not necessarily an euclidian spanner.

The time and message complexity of the API algorithm is the same as for the XTC algorithm.

\subsection{Simulation results}

In this section, we present the simulation results using the metrics presented in Section 3.

Not surprisingly, the LISE algorithm generates a graph that has the lowest maximum edge coverage, since it aims to minimize that specific measure (see Figure 3). The XTC algorithm removes a large portion of edges, including edges with high coverage. The API algorithm tries to reduce the interference for every edge in the network in order to reach a low average interference rather than focusing on the worst case. Combined with the fact that the XTC graph is the sparsest graph, this means that the API algorithm performs worst in this case.

However, considering the average edge interference in Figure 4 , the graph produced by the LISE algorithm has much higher interference than the other two algorithms. This is not surprising, since the average edge interference is defined as the total edge interference divided by the number of nodes. More edges will therefore result in higher interference, and API and XTC both produce sparser graphs than LISE does. 


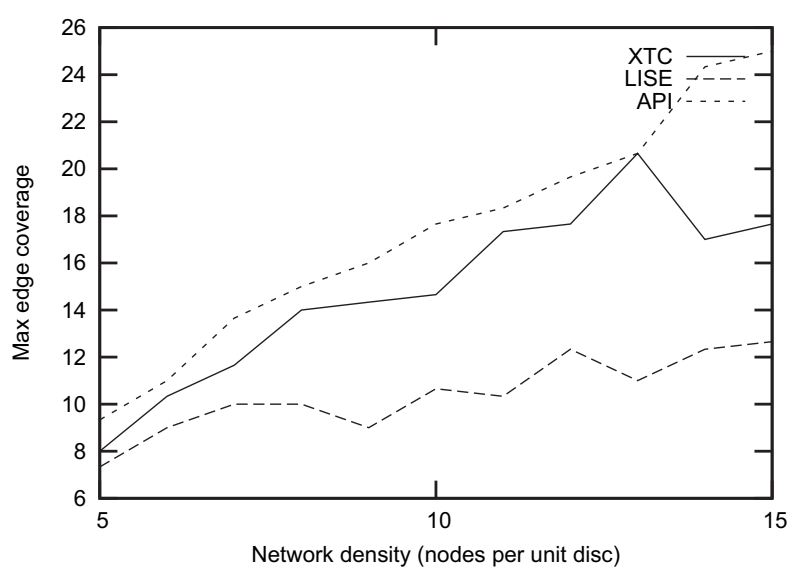

Figure 3: The maximum edge interference.

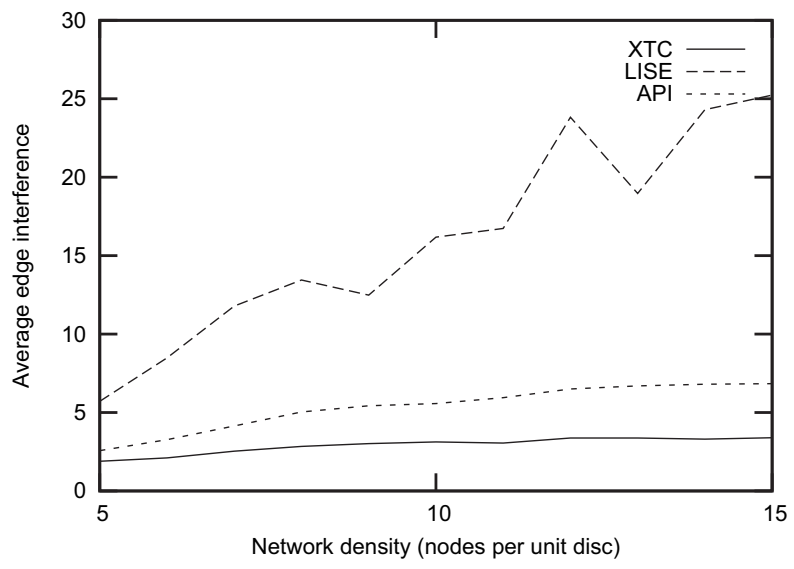

Figure 4: The average edge interference.

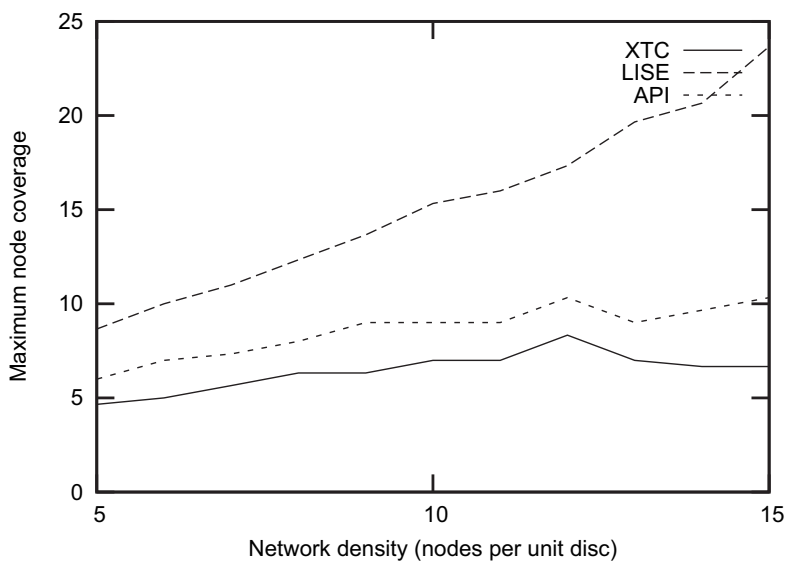

Figure 5: The maximum node coverage.

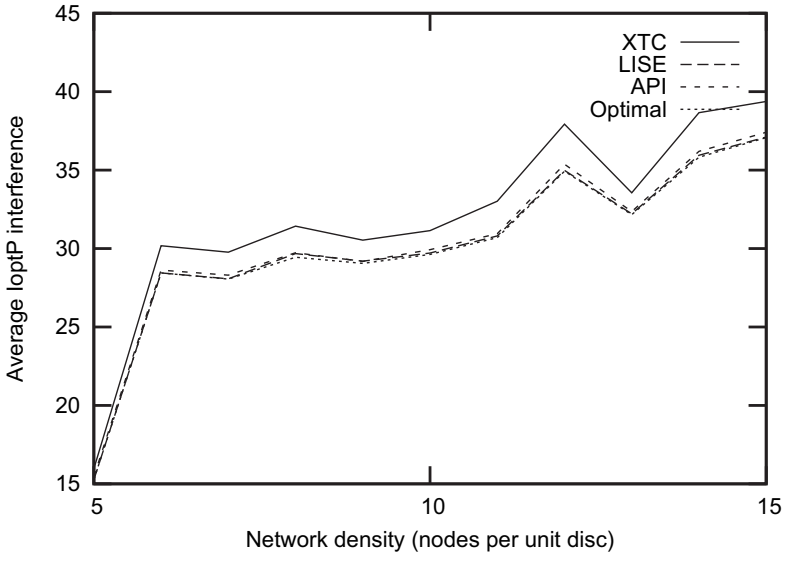

Figure 6: The average interference-optimal path interference.

Considering the maximum node coverage metric (Figure 5 ), it also seems that the sparser graphs have a lower interference, with XTC performing best. But since it is the sparsest graph, it loses some of the interference-optimal paths.

Using the new interference metrics, we see that the API algorithm generally gives the best results. When looking at average path interference with respect to interferenceoptimal paths (Figure 6) we see that both the LISE and the API algorithm preserves almost all interference-optimal paths: the optimal results, which we get when no edges have been removed from the original graph, are almost identical to the results for the two algorithms. In comparison, the results are clearly worse for the XTC algorithm. This is related to the fact that XTC produces the sparsest graph.

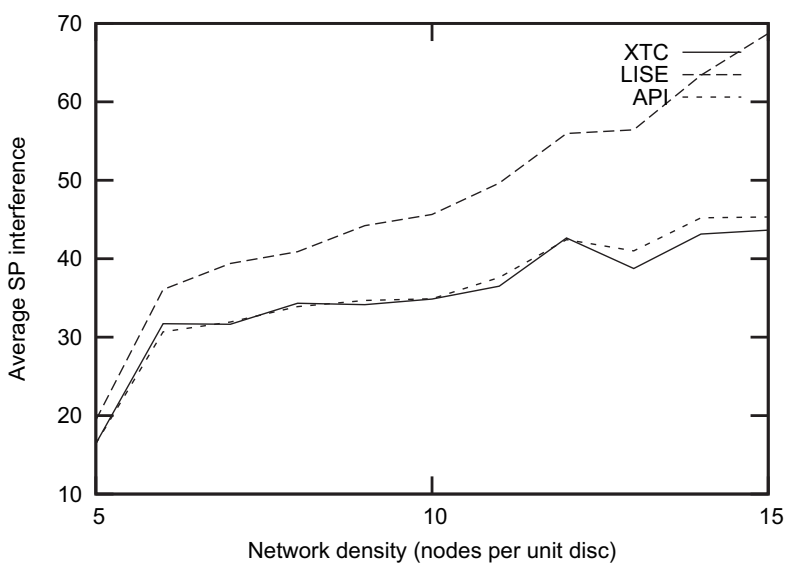

Figure 7: The average shortest-path interference.

When looking at the average path interference with respect to the shortest path (Figure 7), API and XTC give a lower interference than LISE. Also, using the maximum interference difference metric (Figure 8), the API graph has lower interference than both LISE and especially XTC. This means that in the topology resulting from our algorithm, it 


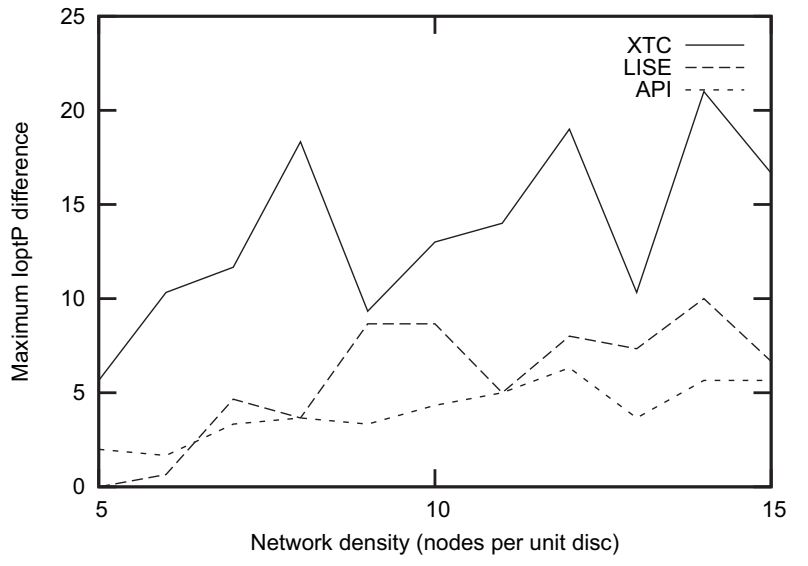

Figure 8: The biggest difference between interference in a interference-optimal path compared to the interference-optimal path in the original topology.

is possible to find paths that are not much worse than the interference-optimal paths in the original graph.

\section{CONCLUSIONS}

In this paper we study how to measure the amount of interference in a wireless network. In contrast to most of the related work we look at the interference of entire paths instead of interference of individual edges or nodes. We propose three new interference metrics that aim to reflect the interference of the entire network.

We also propose a new topology control algorithm, that is shown to produce an energy-spanning graph as a result, while reducing the interference according to previously established interference metrics. We compare it with previously suggested topology control algorithms on randomly generated graphs of various density. We see that sparser graphs generally perform better with respect to the average edge interference and maximum node coverage metrics, but perform worse with respect to metrics based on the interference-optimal paths.

Out of the three implemented algorithms, the API topology gives bad results in the sense of the max edge interference, and less than optimal with respect to the max node interference metric. But it performs better in the case of average edge interference, where its results are close to those of the XTC algorithm, and far better than LISE. This metric is more important for the network communication since a majority of the paths retain a low level of interference.

The average path interference of the API topology matches the better of XTC and LISE for both interferenceoptimal and shortest paths.

Finally, we see that the interference-optimal paths produced by the API algorithm have an interference that always stays close to the interference of the interference-optimal paths in the original topology. For the LISE and especially the XTC algorithm, the worst-case difference is much larger.

\section{REFERENCES}

[1] P. Bose amd L. Devroye, W. S. Evans, and D. G. Kirkpatrick. On the spanning ratio of gabriel graphs and $\beta$-skeletons. In Proceedings of the $5^{\text {th }}$ Latin American Symposium on Theoretical Informatics, 2002.

[2] F. Meyer auf de Heide, C. Schindelhauer, K. Volbert, and M. Gruenewald. Energy, congestion and dilation in radio networks. In Proceedings of the $14^{\text {th }}$ Annual ACM Symposium on Parallel Algorithms and Architecture (SPAA), pages 230-237, 2002.

[3] M. Burkhart, P. von Rickenbach, R. Wattenhofer, and A. Zollinger. Does topology control reduce interference? Proceedings of the $5^{\text {th }}$ ACM Int. Symposium on Mobile Ad-hoc Networking and Computing (MobiHoc), pages 9-19, 2004.

[4] K. R. Gabriel and R. R. Sokal. A new statistical approach to geographic variation analysis. Systematic Zoology, 18:259-278, 1969.

[5] L. Hu. Topology control for multihop packet radio networks. In IEEE Trans. on Communications 41(10), 1993.

[6] L. Iannone, R. Khalili, K. Salamatian, and S. Fdida. Cross-layer routing in wireless mesh networks. In $1^{\text {st }}$ International Symposium in Wireless Communication Systems, 2004.

[7] K. Moaveni-Nejad and X. Li. Low-interference topology control for wireless ad hoc networks. Ad Hoc E Sensor Wireless Networks: an International Journal, 2004.

[8] R. Ramanathan and R. Rosales-Hain. Topology control of multihop wireless networks using transmit power adjustment. Proceedings of the $19^{\text {th }}$ Annual Joint Conference of the IEEE Computer and Communications Societies (INFOCOM), pages 404-413, 2000.

[9] V. Rodoplu and T. Meng. Minimum energy mobile wireless networks. In the 1998 IEEE International Conference on Communications, ICC'98, pages 1633-1639, June 1998.

[10] G. Toussaint. The relative neighborhood graph of a finite planar set. Pattern Recognition, 12:261-268, 1980.

[11] P. von Rickenbach, S. Schmid, R. Wattenhofer, and A. Zollinger. A robust interference model for wireless ad-hoc networks, 2005.

[12] Y. Wang and X. Li. Localized construction of bounded degree and planar spanner for wireless ad hoc networks. Proceedings of the 2003 joint workshop on Foundations of mobile computing, pages 59-68, 2003.

[13] R. Wattenhofer and A. Zollinger. XTC: a practical topology control algorithm for ad-hoc networks. Technical report, Technical report 407, Department of computer science, ETH Zurich, 2003. 